\title{
8 Das Kollegium der Quindecimviri sacris faciundis
}

Dieses Kapitel ist den Mitgliedern des Kollegiums der Quindecimviri sacris faciundis gewidmet, das die Säkularspiele des Jahres I7 v. Chr. ausrichtete. Dabei wird nur ganz am Rande die Funktion und Geschichte dieses Kollegiums dargestellt und ausführlicher auf seine Zusammensetzung zur Zeit der Spiele und die sich daraus ergebende Bedeutung eingegangen.

Die Geschichte des Kollegiums ist in mehreren Abhandlungen und Religionsgeschichten dargestellt. ${ }^{.}$Seine Hauptaufgabe bestand in der Auslegung und Verwahrung der sibyllinischen Bücher und der Einführung fremder Kulte in Rom. In Bezug auf die sibyllinischen Orakel oblag ihnen zwar die Deutung der Orakel, ebenso wichtig war aber, dass sie die Maßnahmen anordneten und zur Durchführung bringen mussten, die die vorangegangene Interpretation des Orakels für nötig erklärt hatte, damit drohendes Unheil abgewendet werden konnte. ${ }^{2}$ Diese wichtige Aufgabe wird auch aus den inschriftlichen Akten der Säkularspiele ersichtlich, allerdings bleibt vollständig im Dunkeln, welcher Art die portenta waren, die vor der Säkularfeier eine Befragung der sibyllinischen Bücher nahegelegt hatten. ${ }^{3}$ Die Quindecimviri sind mehrfach im ersten Teil der Inschrift erwähnt, wo es um die vorbereitenden sakralen Handlungen wie Gebete, Verteilung von Reinigungsmaterial an die Bevölkerung und Annahme von Getreidespenden der Bevölkerung geht, außerdem als Beschlussfassende der verschiedenen Edikte, die den Ablauf der Feier regelten. ${ }^{4}$ Ihre nicht geschlossene Beteiligung an den Riten der eigentlichen Feier wie Opfern und Gebeten vom I. bis 3. Juni und an den ludi honorarii vom 5. bis I2. Juni ist ebenfalls selbstverständlich.' Die eigentliche Durchführung der Opfer und das Sprechen der Gebete wurde vollständig von Augustus und Agrippa übernommen. Die fünf magistri des Kollegiums waren: Augustus, C. Sentius Saturninus, M. Claudius Marcellus, M. Fufius Strigo und D. Laelius Balbus. ${ }^{6}$ Sie hatten an den Opfern sicher ebenfalls Funktionen, sind aber nicht namentlich erwähnt.7 Das augusteische commentarium enthält nur Angaben über die Anwe-

I Wissowa (I9I2) 534ff.; Abaecherli Boyce (I938); Hoffman Lewis (I952); Latte (I960) i6of., 397f.; Radke RE XXIV (1963) i I14-I I48 s.v. Quindecimviri; BNP (I998) 202-203 (zum Kollegium der ludi saeculares von i7 v. Chr.).

2 Wissowa (I912) 539 mit den entsprechenden Quellen; Radke (1963) I I 8 ff.

3 Einzig Zosimus 2, 4, 2 enthält einen kurzen Hinweis darauf; das inschriftliche commentarium hat vielleicht in Z. 52 darauf Bezug genommen.

4 C 5-I 4 ; Z. 29; 43 ff.; 64 ff.; I Ioff.; I 55 ff.

5 So zeigt die vollständig erhaltene Liste von Z. I 50-1 52, dass an den Riten des letzten Tages nur I9 von den insgesamt 2 I Mitgliedern des Kollegiums anwesend waren.

6 CIL I I $^{2}$ p. 29 (Fasti Capitolini), nachträglich eingefügt am linken Rand, unterhalb der Namen der Konsuln des Jahres I 3 n. Chr.; vgl. Pighi (I94I) 42.

7 Zos. 2, 5, 3 erwähnt die Beteiligung der Quindecimviri nur für die zahlreichen Opfer von je neun Schafen und Ziegen in der ersten Nacht. 
senheit der Quindecimviri nach den Riten der Tage, sicherlich weil dieselben Teilnehmer der nächtlichen Riten ohne Pause in den frühen Morgenstunden die Riten des Tages in der Stadt durchführten. Niemals ist das Kollegium in seiner Gesamtheit anwesend, am letzten Tag - und damit auch in der vorangegangenen Nacht - ist allerdings die Teilnahme von I9 der insgesamt 2I Quindecimviri an den Opfern für Apollo und Diana und dem anschließenden Vortrag des carmen saeculare erwähnt. ${ }^{8}$ Dies deutet darauf hin, dass der letzte Tag der ludi saeculares den abschließenden Höhepunkt bildete.

Bis in das Jahr I7 v. Chr. hatte das Kollegium schon verschiedene Umformungen erfahren, vor allem das stetige Anwachsen von Mitgliedern von ursprünglich zwei bis auf eine Zahl von etwa zwanzig Mitgliedern. Diese Vergrößerungen des Kollegiums waren ursprünglich durch die wachsende Zahl fremder Kulte in Rom nötig geworden und hatten dem Kollegium zu einem Prestigegewinn verholfen, der am Anfang des ersten vorchristlichen Jahrhunderts zu einem Stillstand gekommen war. ${ }^{9}$ Mit der Vergrößerung von zehn auf fünfzehn Mitglieder unter Sulla lässt sich in diesem wie in den anderen Priesterkollegien Roms die politische Bedeutung der Besetzung von Priesterämtern verfolgen. Durch die Neuerschließung von sibyllinischer Literatur nach dem Verbrennen der alten Sammlung des Kapitols im Jahr 83 v. Chr. scheint das Kollegium eine wichtige neue Aufgabe gefunden zu haben. Unter Augustus wurde eine Redaktion der sibyllinischen Bücher durchgeführt, die darauf abzielte, die Bücher vom Verdacht der Manipulation und einer möglichen Instrumentalisierung zu befreien. An diesen Maßnahmen war das Kollegium der Quindecimviri beteiligt, indem einige seiner Mitglieder die Bücher eigenhändig abschrieben. ${ }^{\circ}$ Die andere wichtige überlieferte Funktion des Kollegiums war die Ausrichtung der Säkularspiele, die aufgrund eines sibyllinischen Orakels angeordnet waren, somit den Quindecimviri oblagen.

In der Inschrift sind mehrere Listen von Mitgliedern des Kollegiums erhalten, die in Untersuchungen über die Zusammensetzung von Priesterkollegien des Prinzipats herangezogen wurden. ${ }^{11}$ Eine Untersuchung der Mitglieder des Kollegiums führt zu Aufschlüssen über die Bedeutung dieser Priesterschaft zur Zeit der Säkularspiele, das heißt zu einer Zeit, als in der römischen Religion zwar nicht ein Wandel, aber doch eine Neuorganisation vollzogen wurde. Im Folgenden werden zuerst die in der Inschrift erhaltenen Listen vorgestellt, dann die einzelnen Mitglieder des Kollegiums identifiziert. Aus den erhaltenen Listen wird das gesamte Kollegium rekonstruiert und abschließend einige Rückschlüsse über diese Art von Priesterkollegium und seine Zusammensetzung gezogen.

8 Z. I5O-I 52.

9 Abaecherli Boyce (1938) i7off. Boyce stellt die unterschiedlichen Phasen des Kollegiums besonders in seiner Konkurrenz zu den haruspices dar.

Io Cass. Dio 54, I7, 2 und Suet. Aug. 3 I (zur Neuredaktion).

i I Gagé (1931); Hoffman Lewis (1952); Hoffman Lewis (1955); Scheid (1978). 
Die verschiedenen Listen enthalten folgende Namen:

I. C s-8: [ ‥ Imp. Caesar Aug]VSTVS MAG(ister) XVVIR(orum) S(acris) F(aciundis) CVM COLLEGIS L. CENSORINO |[ ․ C. Norbano Fl]ACCO, M. COCCEIO NER-

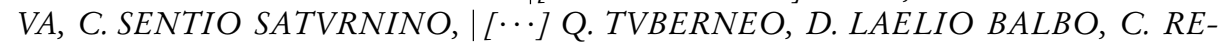
BILO $\mid[\cdots]$

Über die Funktion dieser Liste lässt sich wegen des fragmentarischen Textzustandes nichts sagen. Es ist auffällig, dass Agrippa in dieser Liste nicht genannt ist, da er sonst immer direkt nach Augustus in Aufzählungen erscheint. Somit handelt es sich nicht um eine einführende Vorstellung des gesamten Kollegiums, sondern eher um die Anwesenheitsliste der Quindecimviri an einem Anlass vor der Säkularfeier, wahrscheinlich in der ersten Hälfte des Jahres I 8 v. Chr., als Agrippa von Rom abwesend war. ${ }^{\text {I2 }}$ Die Länge der Liste weist darauf hin, dass das Kollegium fast vollzählig gewesen sein muss.

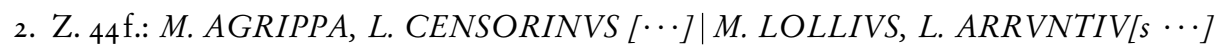

Hier handelt es sich um das Gremium, das die folgenden organisatorischen Beschlüsse für die künftige Säkularfeier gefällt hat. Die Aufzählung beginnt in Z. 44 mit Agrippa und scheint sich über mindestens eine vollständig gefüllte Zeile und eine unbestimmt gefüllte Zeile erstreckt zu haben.

3. Z. I07: AD ATALLAM FVERVNT CAESAR, AGRIPPA, SCAEVOLA, SENTIVS, LOLLIVS, ASINIV[s G]ALLVS, REBILVS.

Bei dieser Liste handelt es sich um die anwesenden Quindecimviri an den Riten der ersten Nacht und des ersten Tages. Die Liste ist vollständig überliefert.

4. Z. I32: AD ATALLAM FVERVNT M. A[grippa ‥ (vacat?)]

Diese unvollständig erhaltene Liste gibt die Anwesenden der Riten des zweiten Tages an. Da Augustus in der Nacht zuvor das Ilithyien-Opfer im Tarentum vollbracht hat, unterscheidet sich die Anwesenheitsliste der Riten der Nacht in diesem Punkt von derjenigen der Riten des Tages. ${ }^{13}$

5. Z. I 50-I 52: XVVIR(i) ADFVERVNT IMP(erator) CA[e]SAR, M. AGRIPPA, Q. LEPIDVS, POTITVS MESSALLA, C. STOLO, C. SCAEVOLA, C. SOSIVS, | C. NORBANVS, M. COCCEIVS, M. LOLLIVS, C. SENTIVS, M. STRIGO, L. ARRVNTIVS, C. ASINIVS, M. MARCELLVS, D. LAELIVS,|Q. TVBERO, C. REBILVS, M[es]SALLA MESSALLINVS.

Diese Aufzählung stellt eine Anwesenheitsliste von I9 Quindecimviri dar, die am Vortrag des carmen saeculare, an den vorangegangenen Riten der dritten Nacht und denen des dritten Tages zugegen waren. Von den insgesamt 2 I Mitgliedern des Kollegiums sind nur L. Censorinus und Cn. Pompeius nicht aufgeführt. Die Liste ist vollständig erhalten.

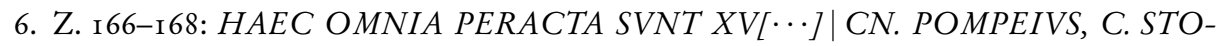

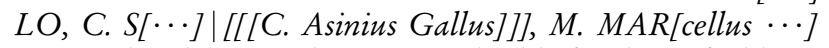

Hier handelt es sich um eine abschließende Aufzählung aller Quindecimviri, die zur Zeit der Säkularspiele dem Kollegium angehörten. ${ }^{14}$ Trotz des fragmentarischen Zustands

I 2 Vgl. Kienast/Eck/Heil (2017) 65; von Juni 20 v. Chr. bis zum Frühjahr I 8 v. Chr. hielt sich Agrippa in Gallien und Spanien auf.

I 3 Vgl. Übersicht über die Riten S. $38 \mathrm{ff}$.

I4 Nach Hoffman Lewis (I952) 290 war die Gesamtzahl des Kollegiums wahrscheinlich 
lässt sich diese Liste vollständig rekonstruieren. Dies ist - wie Hoffman Lewis erstmals gezeigt hat - durch die Kombination der verschiedenen vorangehenden Listen der Inschrift und durch das Erkennen der Prinzipien, nach denen die Seniorität der Mitglieder erstellt wurde, möglich. ${ }^{15}$ Dabei kommt sie zu dem Ergebnis, dass sich die Seniorität der Quindecimviri nicht durch ihren cursus honorum, sondern durch das Jahr ihres Eintritts in das Kollegium ergab. ${ }^{16}$ Es zeigt sich, dass die verschiedenen zum Teil zerstörten Listen die erhaltenen Namen immer in der gleichen Reihenfolge nennen. Folgende Übersicht soll dies verdeutlichen. Die Anwesenheitsliste nach den Riten des zweiten Tages (4. Liste) wurde nicht übernommen, weil dort nur der Name von Agrippa als gesichert gelten kann.

$\begin{array}{lllll}\text { C } 5-8 & 44 \mathrm{f} & \text { I07 } & \text { I } 50-I 52 & \text { I66-I68 }\end{array}$

\begin{tabular}{lll}
\hline Imp. Caes. Aug. & & I. Caesar \\
& M. Agrippa & 2. Agrippa \\
L. Censorinus & L. Censorinus &
\end{tabular}

Scaevola

C. Norban. Fl]accus

M. Cocceius Nerva

C. Senti. Saturninus

$$
\text { M. Lollius }
$$

Sentius

Lollius

L. Arruntius

Q. Tubero

D. Laelius Balbus

C. Rebilus

Asinius Gallus

Rebilus
I. Imp. Caesar
2. M. Agrippa
3. Q. Lepidus
4. Potitus Messalla
5. C. Stolo
Cn. Pompeius
C. Stolo
6. C. Scaevola
7. C. Sosius
8. C. Norbanus
9. M. Cocceius
ı. M. Lollius
I I. C. Sentius
I 2. M. Strigo
I3. L. Arruntius
I4. C. Asinius
I 5. M. Marcellus
I6. D. Laelius
I7. Q. Tubero
I8. C. Rebilus
19. Messalla
Messallinus
C. S[?
[[...]]
M. Mar[cellus

Unstimmigkeiten ergeben sich - wie sie sich schon Mommsen nicht erklären konnte $^{\mathrm{I} 7}$ - in der unterschiedlichen Reihenfolge von Sentius und Lollius in den

noch höher als 21, die sich aus der Liste der Zeilen I66-I68 ergibt. Zur Gesamtzahl des Kollegiums vgl. S. 232.

is Hoffman Lewis (1952) 289-294.

I6 Hoffman Lewis (I952) $289 \mathrm{f}$.: Ein Ausgangspunkt der Überlegung war, dass in der Liste von Z. I 50 ff. einige Quindecimviri, die noch nicht Konsul gewesen waren, vor Konsularen stehen, z. B. C. Licinius Calvus Stolo und C. Mucius Scaevola vor C. Sosius und den folgenden; ein zweiter Ausgangspunkt der Überlegung war, dass im Jahr I7 v. Chr. der sehr junge Quindecimvir M. Valerius Messalla Messallinus noch gar nicht in irgendeiner Weise politisch hervorgetreten war, seine Zugehörigkeit zum Kollegium jedoch auch bei Tibull 2, 5, I7f. bezeugt ist. Schon früher hatte Taylor gezeigt, dass auch andere Priesterschaften in diesem Sinne hierarchisch organisiert waren, Taylor (I942) 39If. Damit wurde eine Vermutung von Marquardt (I884) 243, Anm. I und von Wissowa (I9I2) 50I, Anm. 2 bestätigt.

I7 Mommsen (I89I) 243 (586f.); Mommsen ging davon aus, dass das Jahr der Konsulwürde das Senioritätskriterium darstellte, weswegen er sich die unterschiedliche Rei- 
Listen der Zeilen ro7 und I 5 I, ebenso für D. Laelius Balbus und Q. Tubero in Z. C 7 und I 5 If. Unklar ist auch die Seniorität am Anfang der Liste von L. Censorinus, Q. Lepidus und Potitus Messalla.

Um die von Hoffman Lewis aufgestellte Senioritätsliste nachzuvollziehen, ist es nicht nötig, die Laufbahn der einzelnen Quindecimviri bis ins Detail zu rekonstruieren, was aufgrund der lückenhaften prosopografischen Daten allerdings auch nicht für alle Mitglieder möglich ist. Ich werde mich im Folgenden darauf beschränken, die Quindecimviri nur in Hinsicht auf die Fakten, die für ihren Eintritt in das Kollegium von Bedeutung sind, zu charakterisieren, vollständige Angaben finden sich in den einschlägigen Handbüchern. ${ }^{18}$ Eine umfassende Darstellung der einzelnen Quindecimviri und der älteren Literatur gibt Hoffman Lewis in ihrer Untersuchung über die Priester in julisch-claudischer Zeit. ${ }^{19}$

I. Imperator Caesar Augustus (als erster Quindecimvir in den Listen der Zeilen C 5, I07, I 50 genannt, als Inhaber der tribunicia potestas Z. 52 f., als Opfernder in Z. I03, i Isf., I35 und I39, ergänzt i66)

Über seinen Eintritt in das Kollegium sind wir durch eine Münzabbildung, die auf das Jahr 37 v. Chr. datiert ist, unterrichtet. ${ }^{20}$ Das bedeutet, dass sein Eintritt in das Kollegium spätestens für dieses Jahr anzusetzen ist, wahrscheinlich aber noch früher, eventuell stellt das Priesteramt einen Teil der Erbschaft Caesars dar, dessen Quindecimvirat Augustus gleich nach Caesars Tod übernommen haben könnte. ${ }^{21}$ Es ist nicht eindeutig, ob Augustus und Agrippa die Listen der Zeilen ro $\mathrm{f}$. und I soff. anführen, weil sie Inhaber der tribunicia potestas waren, oder ob sie auch die dienstältesten Mitglieder des Kollegiums der Quindecimviri waren. ${ }^{22}$ Auch wenn beide nicht die dienstältesten Mitglieder des Kollegiums waren, scheinen sie eine Sonderstellung innerhalb des Kollegiums gehabt zu haben, denn sie sind die einzigen Mitglieder des Kollegiums, die Gebete sprechen und Opfer durchführen. Worauf diese Sonderstellung genau gründete, wird in keiner Quelle thematisiert. Scheid vermutet, dass die Mitglieder der kaiserlichen Familien zu den wohl I9 zählenden Mitgliedern des Kollegiums zusätzlich gezählt wurden, was ebenfalls auf eine Sonderstellung hinweist, aber die oben genannte Tatsache auch

henfolge von Sentius und Lollius in den Z. 107 und I I und auch die grundsätzliche Reihenfolge der Quindecimviri nicht erklären konnte.

I8 RE; PIR; Syme (I986).

I9 Hoffman Lewis (I955) $48 \mathrm{ff}$. Rüpke (2007) bietet keine neuen Zusammenhänge.

20 Grueber CRRBM (I9IO) II, 4I5; von einem Eintrittsdatum zwischen 37 und 35 v. Chr. geht Kienast/Eck/Heil (2017) 54 aus.

2 I Cass. Dio 42, 5 I, 4 belegt das Quindecimvirat für Caesar.

22 Hoffman Lewis (1952) 292 und (1955) 88 geht mit Gagé (1935) I 20 davon aus, dass sich die Angabe collega M. Agrippa in RG 22, 2, in denen Augustus die Ausrichtung der Säkularspiele erwähnt, auf das gemeinsame Priesteramt und nicht auf die tribunicia potestas bezieht. 
nicht erklärt. ${ }^{23}$ Augustus und Agrippa sind vor Censorinus genannt, der noch vor den beiden in das Kollegium eingetreten war. Auch dies beweist die Sonderstellung von Augustus und Agrippa in der Seniorität, die nicht auf dem üblichen Kriterium des Eintritts in das Kollegium beruhte, sondern vermutlich allein auf der Tatsache, dass beide Inhaber der tribunicia potestas waren.

2. M. (Vipsanius) Agrippa (als Quindecimvir in den Listen der Zeilen 44, I07, I 5o; als Inhaber der tribunicia potestas in Z. 53; als Opfernder in Z. 104, I 20, I 32, I39; als Startgeber von Wagenrennen in Z. I65, ergänzt I66)

Der Eintritt Agrippas in das Kollegium wird ähnlich früh wie für Augustus vermutet, das heißt, dass auch er bereits Ende der vierziger Jahre Mitglied des Kollegiums gewesen sein könnte. ${ }^{24}$

3. L. Marcius Censorinus (Z. 44, ergänzt I66)

Es handelt sich um den consul ordinarius des Jahres 39 v. Chr., der im Jahr I7 v. Chr. ein mindestens siebzigjähriger Greis gewesen sein muss. ${ }^{25}$ Als entschiedener Anhänger Caesars und einer der beiden Freunde Caesars, die ihn vor seinen Mördern vergeblich schützen wollten, machte Marcius Censorinus in den Jahren nach Caesars Tod eine steile Karriere, die nur während des vorübergehenden Machtgewinns der Senatspartei durch seine Ächtung als Staatsfeind aufgehalten wurde. Nach Antonius' Niederlage gelang es Marcius Censorinus, gute Beziehungen auch zu Augustus aufzubauen. Da er nach Agrippa an erster Stelle genannt ist, muss er von allen Quindecimviri das Mitglied mit dem frühesten Eintrittsdatum in das Kollegium sein, das heißt mit der höchsten Seniorität. Es liegt nahe, anzunehmen, dass der Eintritt in das Kollegium vielleicht noch zu Caesars Zeiten erfolgte, als Caesar nach 49 v. Chr. Priesterämter auch für Nachkommen von Mariusanhängern öffnete. ${ }^{26}$ Damit gehört er zu denjenigen Quindecimviri, die noch nicht von Augustus persönlich optiert wurden. In diesem Sinne stellt er eine alte Generation von Quindecimviri dar.

4. Q. (Aemilius) Lepidus (Z. I 50, ergänzt I66)

Er ereichte das Konsulat als consul ordinarius im Jahr 2 I v. Chr. ${ }^{27}$ Sein Eintritt in das Kollegium muss aber bereits vorher stattgefunden haben, also vor der Zeit seiner größten politischen Erfolge. Ein Priesteramt vor allen anderen politischen Ämtern ist für das Mitglied einer patrizischen Familie durchaus üblich. ${ }^{28}$ Andernfalls könnte seine hohe Seniorität nicht erklärt werden, die er in der Liste von

23 Scheid (1978) 618f.

24 Die Mitgliedschaft Agrippas wird in CIL IX 262, bei Cass. Dio 54, I9, 8 und RG 22, 2 bezeugt. Allerdings fehlt in CIL IX 262 die Konsultitulatur, was für ein Innehaben des Quindecimvirats vor 37 v. Chr. spricht. Vgl. Hoffman Lewis (I955) 48.

25 Münzer RE XIV, 2 (I930) I 554 f. s. v. Marcius Nr. 48; PIR V², I78, Nr. 223.

26 Hoffman Lewis (1955) 88.

27 v. Rohden, RE I (I 893) Sp. 563 f. s. v. Aemilius Nr. 79 (evtl. identisch mit Nr. 78); PIR $\mathrm{I}^{2}, 63$ f., Nr. 376 .

28 Mommsen RStR II, I (I 887) 32. 
Z. I 50 offensichtlich hat, obwohl er sein Konsulat erst acht Jahre später als der nach ihm genannte Potitus Messalla innehatte.

5. (M.? Valerius) Potitus Messalla (Z. I 50, I 54, ergänzt I66)

Rückte als Suffektkonsul im Jahr 29 v. Chr. nach. ${ }^{29}$ Auf einer Inschrift (möglicherweise seinem Grabstein) ist er als Prokonsul von Asien und Legat des Augustus erwähnt. ${ }^{\circ}$ Sein Eintritt in das Kollegium ist ungewiss.

6. Cn. Pompeius (Z. I67)

Er war wahrscheinlich Suffektkonsul im Jahr 3 I v. Chr. ${ }^{31}$ Man hält ihn für identisch mit Cn. Pompeius der Arvalinschriften. ${ }^{32}$ Hoffman Lewis vermutet, dass er aufgrund seiner hohen Seniorität im Kollegium Caesarianer war und deswegen sein Priesteramt schon sehr früh erhalten hatte. ${ }^{33}$

7. C. (Licinius) Stolo (Z. I 50, I67)

Über dieses Mitglied des Kollegiums ist außer seiner hier erwähnten Mitgliedschaft nichts bekannt. Infolge der Stellung muss auch er eher früh eingetreten sein. ${ }^{34}$

8. C. (Mucius) Scaevola (Z. I07, I 50, ergänzt I67)

Ebenso liegen über Scaevola keine anderen Zeugnisse vor als die inschriftlichen der ludi saeculares. ${ }^{35}$

9. C. Sosius (Z. I 50, ergänzt 167 )

Es handelt sich um den bekannten Parteigänger des Antonius und Flottenbefehlshaber bei Actium, der besonders durch Münzprägungen und zahlreiche literarische Zeugnisse bekannt ist. ${ }^{36}$ Im Jahr 32 v. Chr. war er Konsul. Nach der Schlacht bei Actium kam es wieder zu einer Verständigung mit Augustus. Bekannt ist das Engagement des Sosius beim Neuausbau Roms durch die Restaurierung des alten Apollotempels in der Nähe des Marcellustheaters, der seit der aufwendigen Restaurierung in den Jahren 30-28 v. Chr. als templum Apollinis Sosiani bekannt war. Seine Mitgliedschaft im Kollegium der Quindecimviri bestand sicher schon vor seiner Statthalterschaft Syriens, die er 38 v. Chr. antrat, ${ }^{37}$ sie ist außerdem durch eine Münze bezeugt. ${ }^{38}$

29 Hanslik, RE XV (I955) i65f. s. v. Valerius Nr. 267; PIR III, 370, Nr. 94.

30 CIL VI 37075, dort als Quindecimvir erwähnt.

3 I Miltner, RE XXI, 2 (I952) 2055 s. v. Pompeius Nr. 7; PIR III ${ }^{2}, 64$, Nr. 446 (eventuell identisch mit Nr. 444, 445, 447).

32 Scheid (I990c) 23 If.; jetzt in Scheid, CFA (1998) I, 6. I0; und 2, I3. I9; (=CIL VI 32338,2023 und 32339).

33 Hoffman Lewis (1952) 293.

34 Groag, RE XIII, I (1926) 236 s. v. Licinius Nr. 44; PIR V², I, 32, Nr. 179.

35 Groag, RE XVI, I (I933) 424 s. v. Mucius Nr. I4; PIR V², 2, 309, Nr. 694.

36 Fluss, RE III, A I (I927) I I76ff. s. v. Sosius Nr. 2; PIR III 253 , Nr. 556.

37 Hoffman Lewis (1952) 293. Ebenso Broughton, Magistrates II (I952) 427.

38 Grueber CRRBM (I9IO) II, 524. Ob sich der auf der Rückseite der Münze abgebildete Dreifuß auf das Kollegium der Quindecimviri bezieht oder ob es sich um ein altes Münzsymbol von Zakynthos handelt, ist umstritten. Die Literatur dazu bei Hoffman Lewis (1952) 29I, Anm. 9. 
ı. C. Norbanus Flaccus (Z. C 6, I s I, ergänzt i67)

Norbanus war wahrscheinlich der Konsul des Jahres 38 v. Chr. ${ }^{39}$ Sowohl Mommsen als auch Groag gehen davon aus, dass es sich bei dem Quindecimvir der ludi saeculares eher um seinen gleichnamigen Sohn, den Konsul des Jahres 24 v. Chr., handelt. $4^{\circ}$ Wenn man jedoch die Stellung des Norbanus Flaccus in der Seniorität des Kollegiums betrachtet, so sind vor und nach ihm Männer erwähnt, die ihre politische Laufbahn und die Annahme wichtiger Ämter in den dreißiger Jahren erlebten. Es handelt sich bei diesen Mitgliedern des Kollegiums um ehemalige Anhänger verschiedener politischer Lager. Da der Vater, ein ehemaliger Caesarianer, sowohl vom Alter, als auch von seiner Laufbahn her besser an diese Stelle der Seniorität passt, ist er als der Quindecimvir der ludi saeculares dem Sohn vorzuziehen.

I I. M. Cocceius (Nerva) (Z. C 6, I s I, ergänzt i67)

Es handelt sich um den ordentlichen Konsul des Jahres 36 v. Chr. ${ }^{4 \mathrm{I}}$ Als ehemaliger Anhänger des Antonius hatte er wahrscheinlich durch Fürsprache seines Bruders Lucius nach der perusinischen Schlacht Verzeihung erhalten. Sein Eintritt in das Kollegium dürfte etwa in die Zeit seines Konsulats fallen.

I 2. M. Lollius (Z. 45, I07, I I I, ergänzt I67)

Mit Lollius beginnt eine Gruppe von Quindecimviri, die ihre politische Karriere im Gegensatz zu den vorher genannten Quindecimviri nicht in den Zeiten der politischen Machtkämpfe nach Caesars Tod gemacht haben, sondern in den Jahren, als sich die Machtposition von Augustus schon gefestigt hatte. ${ }^{42}$ Diese Männer scheinen etwa gleichaltrig wie Augustus zu sein und können zum Teil als ihm nahestehend bezeichnet werden. Lollius erhielt das Konsulat im Jahr 2 I v. Chr. Seine Beziehung zu Augustus wurde durch die in Germanien erlittene Niederlage eines römischen Heeres unter Lollius im Jahr i 7/6 v. Chr. (clades Lolliana) nicht grundsätzlich getrübt. Mit seinem Eintritt in das Kollegium ist wegen seines Alters nicht vor 3 I v. Chr. zu rechnen. ${ }^{43}$

I3. C. Sentius Saturninus (Z. C 6, I07, I s I, ergänzt I67)

Sentius war Konsul des Jahres I9 v. Chr. ${ }^{44}$ Obwohl er ursprünglich zum Lager der Feinde des Augustus gehörte und Sohn eines Proskribierten war, wurde er später ein verlässlicher Helfer des Herrschers. Einen großen Teil des Jahres hielt Sentius sein Konsulat allein, weil der im Orient weilende Augustus das Konsulat abgelehnt

39 Groag, RE XVII, I (I936) I 270 ff. s. v. Norbanus (cos. 38 v. Chr.) Nr. 9a; PIR V² 383 , Nr. I66.

40 Mommsen (I 89I) 24I (585); Groag, RE XVII, I (I936) 932 ff. s. v. Norbanus (cos. 24 v. Chr.) Nr. 9 .

4I Groag, RE IV, I (I900) I3 I s. v. Cocceius Nr. I3; PIR II², 290, Nr. I 224.

42 Münzer, RE XIII, 2, (I927) I 377 ff. s. v. Lollius Nr. I I; PIR V², I, 83, Nr. 3 I I.

43 Hoffman Lewis (1952) 293; ebenso Broughton, Magistrates II (1952) 426, der Lollius in seiner Liste der Quindecimviri des Jahres 3 I nicht aufführt.

44 Groag, RE II A, 2 (I923) is I Iff. s. v. Sentius Nr. 9; PIR III², I99, Nr. 293. 
hatte, und ein Kollege noch nicht gewählt war. Außerdem ist er in den kapitolinischen Fasten als einer der fünf magistri des Kollegiums aufgeführt. ${ }^{45}$ Das bedeutet, dass er innerhalb des Kollegiums eine hervorragende Stellung einnahm, die er nicht aufgrund einer besonders hohen Seniorität innehatte, sondern aufgrund eines besonderen Vertrauensverhältnisses zu Augustus.

I4. M. (Fufius) Strigo (Z. I s I, ergänzt I67)

Über Fufius Strigo ist nur durch den Eintrag in die kapitolinischen Fasten bekannt, dass er einer der fünf magistri des Kollegiums der Quindecimviri war. ${ }^{46}$ Münzer geht davon aus, dass er ebenfalls konsularischen Ranges war, weil neun der vor ihm genannten Quindecimviri und zwei der nach ihm genannten diesen Rang haben. ${ }^{47}$ Dieser Schluss geht davon aus, dass die Seniorität der Quindecimviri in irgendeiner Weise durch ihre politische Laufbahn bestimmt war. Obwohl solche Zusammenhänge für einige Quindecimviri erkennbar sind, kann es aber durchaus möglich sein, dass Fufius Strigo ohne entsprechenden politischen Rang eine hohe Stellung im Kollegium der Quindecimviri innehatte, die er seinen persönlichen Beziehungen zu Augustus verdankte.

I 5. L. Arruntius (Z. 45, is I, ergänzt I67)

Arruntius war Konsul im Jahr $22 \mathrm{v}$. Chr..$^{8}$ Auch er scheint sein Verhältnis zu Augustus nach anfänglichen Schwierigkeiten bereinigt zu haben. Es ist nicht ganz klar, ob er, sein Vater oder beide proskribiert waren, an der Schlacht von Actium nahm er jedoch in wichtiger Funktion teil. ${ }^{49}$ Sein Eintritt in das Kollegium muss etwa in der Zeit zwischen Actium und seinem Konsulat gelegen haben.

I6. C. Asinius Gallus (Z. AB 9, I07, I s I, ergänzt C 7; getilgt I68)

Asinius gehört zu der Gruppe der jüngeren Mitglieder des Kollegiums, die im Jahr I7 v. Chr. noch nicht das Konsulat erreicht hatten. ${ }^{\circ}$ Er erhielt das Konsulat im Jahr 8 v. Chr. Ein unüblicher Karrieresprung belegt sein gutes Verhältnis zu Augustus. $^{\text {I }}$ Persönliche Konflikte mit Tiberius führten dazu, dass sein Name in Z. I68 am linken Rand der Inschrift später getilgt, an weniger exponierter Stelle allerdings belassen wurde. ${ }^{2}$ Über das Datum seines Eintritts in das Kollegium lassen sich keine Angaben machen.

45 Pighi (I94I) 42 oder CIL I². I p 29.

46 Pighi (I94I) 42 oder CIL I'2. P 29.

47 Münzer, RE VII, I (I9I0) 2 I0 s. v. Fufius Nr. I9; PIR III'2, 207, Nr. 5 I 3.

48 v. Rohden, RE II, I (I895) I 262 s. v. Arruntius Nr. 7; PIR I², 220, Nr. I I 29.

49 Vell. 2, 85, 2 erwähnt Arruntius als Befehlshaber des linken Flügels.

so Klebs, RE II, 2 (I896) I 58 ff. s. v. Asinius Nr. I 5 ; PIR I², 245 ff., Nr. I 229.

I So erhielt er ein Prokonsulat von Asien ohne Einhaltung des gesetzlichen quinquennium nach nur zwei Jahren. Auch die Tatsache, dass er sein Porträt auf Münzen prägen durfte, scheint seine priviligierte Stellung zu bestätigen. Klebs, RE a.a.O., Sp. I 585 .

52 Wahrscheinlich war die Heirat mit Vipsania Agrippina nach deren erzwungener Scheidung von Tiberius im Jahr I 2 v. Chr. ein Anlass für diesen Konflikt. Tac. Ann. I, I 2; Cass. Dio 57, 2, 7 . 
I7. M. (Claudius) Marcellus (Aserninus) (Z. I I und fragmentarisch I68)

Hinsichtlich der Identifikation dieses Quindecimvir bestehen Differenzen. Mommsen und Pighi hielten ihn für den Konsul des Jahres 22 v. Chr., ${ }^{33}$ Hoffman Lewis dagegen identifiziert ihn mit einem M. Claudius Marcellus, der ein Sohn des Konsuls von 22 v. Chr. ist. ${ }^{54}$ Folgt man Hoffman Lewis' Überlegungen hinsichtlich der drei Gruppen von Quindecimviri, die sich aus ihrer politischen Laufbahn ergeben, passt der Vater M. Marcellus (cos. 22 v. Chr.) nicht in die mit dem vorangehenden C. Asinius Gallus beginnende dritte Gruppe jüngerer Mitglieder des Kollegiums. Der Vater M. Marcellus müsste aufgrund seines Konsulats und seines Alters zu der zweiten Gruppe von Quindecimviri gehören, die die Mitglieder Lollius, Sentius, Strigo und Arruntius umfasst. Der Sohn M. Claudius Marcellus hatte zur Zeit der Säkularspiele noch kein Konsulat inne, dennoch wird er in den kapitolinischen Fasten als magister des Kollegiums der Quindecimviri genannt. 55 Dies lässt die Vermutung zu, dass auch er, wie die beiden schon erwähnten magistri Sentius und Strigo, in einem besonderen persönlichen Vertrauensverhältnis zu Augustus stand. Auch seine Integration seit früher Kindheit in die ersten Kreise der römischen Gesellschaft sprechen für eine persönliche Beziehung zwischen Augustus und Marcellus. ${ }^{56}$

I 8. D. Laelius (Balbus) (Z. C 7, I s I, ergänzt I68)

Auch Laelius ist in den kapitolinischen Fasten als magister des Kollegiums der Quindecimviri aufgeführt. ${ }^{57}$ Er wird als der ordentliche Konsul des Jahres 6 v. Chr. identifiziert. Weitere biografische Fakten wie ein Aufenthalt als Quaestor pro praetore in Nordafrika vor seinem Konsulat sind nicht gesichert.

I9. Q. (Aelius) Tubero (Z. C 7, I 52, ergänzt I68)

$\mathrm{Da}$ er mit D. Laelius in Z. C 7 und I $\mathrm{I}$ If. in unterschiedlicher Reihenfolge genannt ist, scheint er dasselbe Eintrittsdatum in das Kollegium der Quindecimviri zu haben wie Laelius. Allgemein wird er als der Konsul des Jahres i I v. Chr. identifiziert. ${ }^{58}$ Er scheint als erstes Mitglied seiner Familie ein Konsulat und ein so hohes Priesteramt erlangt zu haben und dies, wie sonst nur für nobiles üblich, schon in jungen Jahren. ${ }^{59}$

53 Mommsens Marcellus (cos. 22 v. Chr.) = PIR II', 2 I 5 , Nr. 926. Pighi hat die Identifikation Mommsens übernommen: Pighi (I94I) 234.

54 Ebenso Syme (i986) 49, Anm. I I. Zum gleichnamigen Sohn des Konsuls von 22 v. Chr.: Groag, RE III, 2 (I 899) 277I s. v. Claudius Nr. 234; PIR II² 2 I 5 , 927.

55 Pighi (I94I) 42 oder CIL I². I p 29.

56 Überliefert ist die Teilnahme des Marcellus (Sohn) am Trojaspiel: Suet. Aug. 43.

57 Pighi (I94I) 42; CIL I². p 29; Angaben zur Person: Miltner, RE XII, I (I924) 4I 5 s. v. Laelius Nr. I5; PIR V², I0, Nr. 47.

58 Hoffman Lewis (1952) 293. Mommsen war sich nicht sicher, ob es sich bei Tubero um den Konsul des Jahres i I v. Chr. handelt; vgl. Mommsen (I892) 240 (584). Auch v. Rohden, RE I, I (I893) 538 s. v. Aelius Nr. I 57 erwähnt das Priesteramt als Quindecimvir nicht, ebenso PIR I'2, 45, Nr. 274.

59 Syme (1986) 57. 
20. C. (Caninius) Rebilus (Z. C 7, I07, I 52, ergänzt ı68)

Es handelt sich um den Suffektkonsul des Jahres I 2 v. Chr., der im Amt starb. ${ }^{60}$ Sein Eintritt in das Kollegium erfolgte wahrscheinlich nicht sehr lange vor den Säkularspielen, da er in der Seniorität der Quindecimviri an vorletzter Stelle steht, wie aus Z. I 52 hervorgeht. Es sind keine weiteren Zeugnisse über seine Laufbahn oder politischen Beziehungen bekannt.

2 I. M. (Valerius) Messalla Messallinus (Z. I 52, ergänzt ı68)

Als letzter der Liste der Quindecimviri erscheint Messalla Messallinus, der das Konsulat im Jahr 3 v. Chr. erlangte. ${ }^{6 \mathrm{r}}$ Er muss zur Zeit der Säkularspiele also noch sehr jung gewesen sein, sein Geburtsjahr wird frühestens auf das Jahr 39 v. Chr., spätestens das Jahr 36 v. Chr. angesetzt. ${ }^{62}$ Dennoch sprechen einige Gründe für seine verfrühte Aufnahme in das Kollegium der Quindecimviri: Das Geschlecht der Valerier ist mit dem Mythos der Säkularspiele eng verbunden, wonach ein Valerier zum ersten Mal anlässlich der wunderbaren Heilung seiner Kinder Spiele ausrichtete, die in der Überlieferung als Vorgänger der späteren Säkularspiele verstanden wurden. ${ }^{63}$ Für spätere Säkularspiele ist die Teilnahme mindestens eines Mitglieds der gens Valeria als Mitglied des ausrichtenden Kollegiums der Quindecimviri überliefert. ${ }^{64}$ Es ist deshalb durchaus denkbar, dass der junge Valerius Messalla Messallinus einerseits die Möglichkeit hatte, als Angehöriger einer patrizischen gens seine Karriere schon früh mit einem Priesteramt zu beginnen und dass sich andererseits im Hinblick auf die künftigen Säkularspiele mit dem Quindecimvirat ein solches - wenn auch etwas früh - für ihn besonders anbot. Damit ist die gens Valeria an den Säkularspielen mit zwei Mitgliedern im ausführenden Priesterkollegium gebührend vertreten. Da sein Priesteramt bei Tibull belegt ist, muss er es noch zu Lebzeiten Tibulls, also vor dem Jahr i 9 v. Chr. erhalten oder in Aussicht gestellt bekommen haben. ${ }^{65}$ Außerdem war seit Potitus Valerius Messalla, dessen Eintritt in das Kollegium schon lange zurücklag, kein Patrizier mehr gewählt worden. ${ }^{66}$

60 Groag, RE III, 2 (I 899) I 479 s. v. Caninius Nr. Io; PIR II², 94, Nr. 39I, Syme (I986) 55, I 53 .

6 I v. Lunzer, RE VIII A, I (I955) I 59 ff. s. v. Valerius Messalla Messallinus Nr. 264; PIR III, 369, Nr. 93 .

62 v. Lunzer RE, I 59.

63 Zos. 2, I, I-3. Val. Max. 2, 4, 5. Über die Verbundenheit der gens Valeria mit dem Marskult und den ludi saeculares vgl. Versnel (I982) 2 I 2 ff. Vgl. Kap. A.6, S. I90f.

64 Vgl. Tabelle S. I7I.

65 Tibull 2, 5, i 7 f.

66 Da Potitus Valerius Messalla sein Priesteramt spätestens zum Zeitpunkt seines Konsulats (29 v. Chr.) - als Patrizier aber wahrscheinlich früher - erhalten hatte, war während mindestens zehn Jahren kein Mitglied einer patrizischen gens in das Kollegium der Quindecimviri aufgenommen worden. 
Die Liste des gesamten Kollegiums in Z. I66-I68 lässt sich nun nach folgenden Überlegungen rekonstruieren: Zwischen dem ersten inschriftlich erhaltenen $\mathrm{Cn}$. Pompeius in Z. 167 und dem Erstgenannten der Liste Imp. Caesar Augustus müssen sich durch eine vergleichende Nebeneinanderstellung mit den Listen von I $50 \mathrm{ff}$. und ${ }_{44} \mathrm{f}$. senioritätsmäßig vier Mitglieder des Kollegiums befunden haben: ${ }^{67}$ M. Agrippa, L. Censorinus, Q. Lepidus und Potitus Messalla. Ich habe mich entschieden, direkt nach M. Agrippa L. Censorinus folgen zu lassen, weil dieser auch in Z. C 5 als erster der auf Augustus folgenden Kollegen genannt ist und weil Censorinus aufgrund seines Alters sehr wahrscheinlich eine außerordentlich hohe Seniorität hatte. Darauf folgen Lepidus und Potitus Messalla, sie lassen sich in der Reihenfolge, wie sie in Z. I 50 vorliegt, ergänzen. Die Ergänzung von Z. I67 folgt ohne Schwierigkeiten der Reihenfolge von Z. I sof., ebenso wie die Ergänzung von Z. $168 .^{68}$

Damit haben wir in Z. I66-168 eine Gesamtdarstellung des Kollegiums erhalten, die erlaubt, weitere Rückschlüsse über die Zusammensetzung des Kollegiums zu ziehen. Die augusteische Restauration des Kollegiums baute auf einem bestehenden Kern von alten Mitgliedern auf, deren Eintritt in das Kollegium zum Teil noch zu Caesars Zeiten oder in den ersten zehn Jahren nach seinem Tod erfolgt war. Zu diesem alten Kern gehört nach Hoffman Lewis folgende Gruppe von elf Quindecimviri: ${ }^{6}$

I. Imperator Caesar Augustus

2. M. Vipsanius Agrippa, cos. ord. 37, 28, 27 v. Chr., trib. pot. I 8 v. Chr.

3. L. Marcius Censorinus, cos ord. 39 v. Chr.

4. Q. Aemilius Lepidus, cos. ord. 2 I v. Chr.

5. Potitus Valerius Messalla, cos. suff. 29 v. Chr.

6. Cn. Pompeius, cos. suff. 3 I v. Chr.

7. C. Licinius Calvus Stolo

8. C, Mucius Scaevola

9. C. Sosius, cos. ord. 32 v. Chr.

Io. C. Norbanus Flaccus, cos. ord. 38 v. Chr.

I I. M. Cocceius Nerva, cos. ord. 36 v. Chr.

Bei dieser Gruppe handelt es sich um Männer, deren politische Laufbahn in den dreißiger Jahren des letzten vorchristlichen Jahrhunderts ihren Höhepunkt erreicht hatte. Sie waren bei den Auseinandersetzungen nach Caesars Tod im Lager der Antoniusanhänger und der Caesarianer engagiert, was den Schluss nahelegt, dass ihr Eintritt in das Kollegium wohl auf das Ausbalancieren der wechselnden Kräfteverhältnisse im Triumvirat zurückzuführen ist.

67 Vgl. S. 230.

68 Z. I 66 erhält mit dieser Ergänzung eine Buchstabenzahl von 83 Buchstaben, für Z. I 67 ergibt sich eine Buchstabenzahl von 80 Buchstaben, Z. I68 war nicht bis an den rechten Rand ausgeschrieben.

69 Hoffman Lewis (I952) 29if. und (I955) $48 \mathrm{ff}$. und $87 \mathrm{f}$. 
Das entscheidende Kriterium zur Abgrenzung einer zweiten Gruppe von Quindecimviri von dieser ersten Gruppe der älteren Quindecimviri ist nicht nur in dem Stand ihrer Laufbahn zu finden, sondern in erster Linie darin, dass die Mitglieder dieser zweiten Gruppe zu einem Zeitpunkt in das Kollegium eingetreten sein müssen, als Augustus schon angefangen hatte, gewisse Strukturen der römischen Religion wieder zu stärken. ${ }^{70}$ Dazu gehörte die Restaurierung der vernachlässigten Tempel und vor allem die Besetzung der lange vakant gebliebenen Priesterämter. ${ }^{71}$ Es ist sinnvoll, die noch später in das Kollegium eingetretenen Quindecimviri mit diesen Maßnahmen in Beziehung zu setzen und ihre Mitgliedschaft als Folge dieser Bestrebungen zu sehen. Allgemein wird das Jahr 29 v. Chr. als der Beginn der sogenannten augusteischen Restauration in politischer und religiöser Hinsicht angesetzt. Für die Besetzung der Priesterämter trifft dieses Datum sicher zu, denn im Jahr 29 v. Chr. wurde Augustus das Recht verliehen, so viele Priester, wie er wünschte, zu ernennen, sogar über die übliche Zahl hinaus. ${ }^{72}$ Auch wenn im Jahr 29 v. Chr. ein mehrere Jahre währender Prozess der Errichtung religiöser Institutionen erst einsetzte, wovon die Ernennung von Priestern nur einen Aspekt darstellt, ist es sinnvoll, seit $29 \mathrm{v}$. Chr. einen Wendepunkt in der Besetzung des Kollegiums der Quindecimviri zu sehen. Wir wissen, dass das Kollegium im Jahr 3 I v. Chr. I6 Mitglieder hatte, demnach muss seine Zahl von Augustus bis zum Jahr I7 v. Chr. um mindestens fünf Mitglieder erhöht worden sein. Es haben also fünf Neuaufnahmen und eventuelle Ersatzaufnahmen stattgefunden. ${ }^{73}$ Deswegen kann man die Mitgliedschaft der zweiten Gruppe von Quindecimviri auf den Einfluss von Augustus allein zurückführen. Zu dieser zweiten Gruppe gehören folgende Mitglieder:

70 Hoffman Lewis hat als zusätzliches Kriterium der Abgrenzung innerhalb dieser zweiten Gruppe von Quindecimviri die Erlangung des Konsulats bis zum Jahre I7 v. Chr. angenommen. Weil die erste Gruppe jedoch auch Mitglieder enthält, für die kein Konsulat bezeugt ist, scheint mir dieses weitere Unterscheidungskriterium nicht sinnvoll. Ich fasse die von Hoffman Lewis (I952) $292 \mathrm{f}$. und (I955) $88 \mathrm{f}$. in zwei Gruppen eingeteilten Quindecimviri deswegen zu einer einzigen zusammen. Hoffman Lewis versteht M. Lollius, C. Sentius Saturninus, M. Fufius Strigo und L. Arruntius als eine Gruppe von Mitgliedern, die im Jahr der Säkularspiele schon Konsulare waren, wogegen die zweite Gruppe mit C. Asinius Gallus, M. Claudius Marcellus, D. Laelius Balbus, Q. Aelius Tubero, C. Caninius Rebilus und M. Valerius Messalla Messallinus das Konsulat erst nach den Säkularspielen erlangte. Auch Radke RE XXIV (1963) s. v. Quindecimviri I 45 f. geht nur von einer Unterscheidung der vor und nach 28 gewählten Quindecimviri aus.

7I Zur Bautätigkeit: Gros (1976)I 5-52; Kolb (1995) 330ff.; Zanker (19973) I Ioff.; zur Besetzung der Priesterstellen: Hoffman Lewis (I 955) 7-23 und I I I-I I 7; Liebeschütz (I979) 62-64; Scheid (I990a) 684-686.

72 Cass. Dio 51, 20, 3 .

73 Scheid (I978) 6I 8. 
I. M. Lollius, cos. ord. 2 I v. Chr.

2. C. Sentius Saturninus, cos. ord. I9 v. Chr.

3. M. Fufius Strigo

4. L. Arruntius, cos. ord. 22 v. Chr.

5. C. Asinius Gallus, cos. ord. 8 v. Chr.

6. M. Claudius Marcellus

7. L. Laelius Balbus, cos. ord. 6 v. Chr.

8. Q. Aelius Tubero, cos. ord. I I v. Chr.

9. C. Caninius Rebilus, cos. suff. I 2 v. Chr.

ı. M. Valerius Messalla Messallinus, cos. ord. 3 v. Chr.

Obwohl ein enger Zusammenhang zwischen Laufbahn und Eintritt in ein Kollegium besteht, zeigt die Zusammensetzung des Kollegiums der Quindecimviri, dass die Erlangung des Konsulats nicht das einzige Kriterium einer Aufnahme war. Für den Angehörigen einer patrizischen gens war es durchaus möglich, lange vor Besetzung des Konsulats in das Kollegium zu gelangen, wie die Beispiele von Q. Aemilius Lepidus und M. Valerius Messalla Messallinus zeigen. ${ }^{74}$ Es ist nicht erstaunlich, dass die neben Augustus fungierenden vier magistri des Kollegiums in der zweiten Guppe von Quindecimviri zu finden sind. Dazu gehören abgesehen von Augustus selbst:

C. Sentius Saturninus

M. Fufius Strigo

M. Claudius Marcellus

D. Laelius Balbus.75

Obwohl wir über die genaue Funktion dieses Amts nicht unterrichtet sind, ist doch auffällig, dass es ganz offensichtlich in keinem Zusammenhang mit der Seniorität eines Quindecimvir steht. Es scheint eher wichtig gewesen zu sein, dass zwischen Augustus und den anderen magistri eine persönliche Beziehung bestanden hat, wie es wenigstens für Sentius Saturninus und Claudius Marcellus vermutet werden kann. Auch aus den inschriftlichen Aufzeichnungen geht in keiner Weise etwas über eine eventuelle Aufgabe der magistri hervor; sie sind in der Liste von C sff. alle genannt, ebenso in der Liste der Zeilen I $50 \mathrm{ff}$. und I66ff. Welche rituellen Aufgaben einzelne magistri während der Zeremonien hatten, ist unbekannt. Es ist aber anzunehmen, dass sie angesichts des gewaltigen organisatorischen Aufwands vor und während der Feier besondere Aufgaben zu erfüllen hatten.

Die genaue Kenntnis des Kollegiums zur Zeit der Säkularspiele erlaubt uns, Rückschlüsse auf seine soziale Durchmischung zu ziehen und Vermutungen über mögliche Aufnahmekriterien anzustellen. Die Namen der fünf magistri des Kollegiums sind fettgedruckt.

74 Mommsen, RStR II, I (I 8873) 32; Taylor (I942) 409; Hoffman Lewis (I952) 293, Anm. I4; Dio Cass. 49, I6, I.

75 Die Namen der magistri sind in den kapitolinischen Fasten eingetragen, vgl. Pighi (I94I) 42 oder CIL I'. I p. 29. 
Patrizische gens:

Gruppe I: Imperator Caesar Augustus, Q. Aemilius Lepidus, Potitus Valerius Messalla

Gruppe 2: M. Valerius Messalla Messallinus

Patrizische gens nach der lex Saenia (29 v. Chr.):

Gruppe r: M. Vipsanius Agrippa, M. Cocceius Nerva

Gruppe 2: C. Sentius Saturninus, L. Arruntius, C. Asinius Gallus, M. Claudius Marcellus, Q. Aelius Tubero

Plebeische gens:

Gruppe r: L. Marcius Censorinus, Cn. Pompeius, C. Licinius Calvus Stolo, C. Mucius Scaevola, C. Sosius, C. Norbanus Flaccus

Gruppe 2: M. Lollius, M. Fufius Strigo, D. Laelius Balbus, C. Caninius Rebilus

Aus dieser Zusammenstellung wird klar, dass sowohl in der ersten wie auch in der zweiten Gruppe der Quindecimviri ein ausgewogenes Verhältnis zwischen Patriziern und Plebejern herrschte. ${ }^{76}$ Von den I I Quindecimviri der ersten Gruppe waren fünf Patrizier, sechs Plebejer, in der zweiten Gruppe sechs Patrizier und vier Plebejer. Allerdings stammen drei der Patrizier der ersten Gruppe aus der alten patrizischen Nobilität, während nur zwei nach der lex Saenia zu den neu ernannten Patriziern nach 29 v. Chr. gehören. Von den sechs Plebejern der ersten Gruppe gehören vier der alten plebejischen Nobilität an (Censorinus, Pompeius, Scaevola und Flaccus), während nur zwei homines novi aus plebejischem Geschlecht vertreten sind (Stolo und Sosius). In der zweiten Gruppe befindet sich unter den insgesamt sechs Patriziern nur einer aus altem patrizischen Geschlecht (M. Valerius Messalla Messallinus), alle anderen gehören zu den neu ernannten Patriziern. Entsprechend gehört keiner der vier Plebejer zu einer alten plebejischen Familie. Dieselbe Ausgewogenheit lässt sich auch bei den vier magistri beobachten: Zwei stammen aus einer patrizischen, zwei aus einer plebeischen Familie. Allerdings sind nur patrizische Geschlechter nach der lex Saenia berücksichtigt.

Als Fazit dieser sozialen Zusammenstellung des Kollegiums ist festzuhalten, dass sich das Kollegium der Quindecimviri besonders nach 29 v. Chr. als ein offenes Kollegium präsentiert.77 Ein Vergleich der beiden Gruppen zeigt deutlich, dass die Vertreter der alten Familien in der ersten Gruppe noch ein Übergewicht haben. Seit der Kontrolle des Kollegiums durch Augustus allein finden wir jedoch fast nur Aufsteiger im Kollegium und außer Valerius Messalla Messallinus keine Vertreter alter Priesterfamilien der Republik mehr. Dies entspricht den Rekrutierungen von Mitgliedern in den anderen großen Priesterkollegien. ${ }^{78} \mathrm{Da}$ das Kol-

76 Dieses Gleichgewicht zwischen Patriziern und Plebejern ist für das Kollegium der Quindecimviri seit der Erweiterung des Kollegiums von einem Duumvirat auf ein Decemvirat im Jahr 367 v. Chr. überliefert. Siehe die entsprechenden Quellen bei Wissowa (1912) 535 .

77 Scheid (1978) $637 \mathrm{f}$.

78 Scheid (1978) 632-639 und (1990a) 701 . 
legium der Quindecimviri wie auch das der angures für Augustus in gewisser Weise eine Vorbildfunktion erfüllte, wie Gagé ausgeführt hat, ist durchaus denkbar, dass Augustus versuchte, über den Einfluss auf diese Kollegien seine Vorstellungen über das Funktionieren einer Priesterschaft zu verdeutlichen, schon bevor er das Amt des pontifex maximus nach dem Tod des Lepidus im Jahr I2 v. Chr. endlich übernehmen konnte..$^{79}$ Die sorgfältige Auswahl der künftigen Mitglieder des Kollegiums gehörte in entscheidendem Maße dazu.

Die Inschrift erwähnt in der abschließenden Aufzählung des gesamten Kollegiums, die - wie wir gesehen haben - vollständig rekonstruierbar ist, die Zahl von 2 I Mitgliedern. Die Meinungen, ob dies die Gesamtzahl aller Mitglieder war, gehen auseinander: Während Hoffman Lewis und Syme eher der Meinung sind, dass es sich bei der Aufzählung von Z. I66ff. nur um diejenigen Mitglieder handelt, die auch tatsächlich an den Feierlichkeiten vom I. bis 3. Juni des Jahres I7 v. Chr. in Rom anwesend waren, und das Kollegium wahrscheinlich noch einige zusätzliche Mitglieder hatte, die zu dieser Zeit nicht in Rom weilten, geht Scheid davon aus, dass mit der Aufzählung die Gesamtzahl des Kollegiums angegeben wird. ${ }^{80}$ Seine These begründet Scheid mit einem Vergleich der Zahl der Quindecimviri der augusteischen und der severischen Säkularfeier: Das augusteische Kollegium bestand aus 19 Mitgliedern plus zwei Mitgliedern aus der Familie des Augustus, nämlich Augustus selbst und Agrippa. Das severische Kollegium bestand nach Scheid ebenfalls aus I9 Mitgliedern plus vier Mitgliedern der kaiserlichen Familie. ${ }^{{ }^{1}}$ Auch wenn dieses Argument wegen der wenig gesicherten Zahl des severischen Kollegiums nicht vollständig überzeugend ist, muss doch wegen der Wichtigkeit des Anlasses davon ausgegangen werden, dass das Kollegium der Quindecimviri vollständig vertreten war. Kein Anlass war in der Geschichte dieses Kollegiums von vergleichbarer Bedeutung, und es erscheint mit Scheid einleuchtend, dass auch Mitglieder, die gewöhnlich von Rom abwesend waren, speziell für diesen Anlass nach Rom kamen. ${ }^{82}$ Ein weiteres Argument liefert die Art der

79 Gagé (I93i) 96f. und roif.

80 Hoffman Lewis (I955) 89; Syme (I986) 49; Scheid (I978) 6r 8 f. und (I990c) I 50.

8 I Pighi (I94I) 240f.; Schumacher (I973) I66.

82 Scheid (1978) 619 führt aus, dass der Zeitpunkt der ludi saeculares Ende Mai/Anfang Juni auch den Statthaltern von Provinzen die Möglichkeit bot, etwas früher als üblich in Rom einzutreffen in Anbetracht dieses besonderen Anlasses. Dagegen Schumacher (1978) 703 f.: Schumacher glaubt, dass ein verfrühtes Verlassen der Statthalter ihres Postens dem Prinzip römischer Reichverwaltung widerspreche. Seine Annahme, dass das Kollegium der Quindecimviri im Jahr 17 v. Chr. doch einige in der Inschrift unerwähnte Mitglieder hatte, stützt Schumacher auf die Überlieferung des Namens eines weiteren Quindecimvir, dem Konsul des Jahres i9 v. Chr., M. Vinicius (Degrassi, Inscr. Ital. XIII 3 p. 75 ff. = Dessau III, 2 8965), der aber nicht in den inschriftlichen Aufzeichnungen der ludi saeculares begegnet. Allerdings ist nicht gesichert, ob Vinicius im Jahr I7 v. Chr. schon Quindecimvir war, vom Alter her wäre seine Mitgliedschaft möglich (Radke RE XXIV (I963) s. v. Quindecimviri I I46). Vgl. 
Aufzählung des Kollegiums am Ende der Inschrift. Diese Aufzählung ist keine Anwesenheitsliste bei bestimmten Riten der Feier. Sie hat keine andere Funktion, als die Mitglieder des Kollegiums der Quindecimviri zur Zeit der Säkularspiele festzuhalten. Die Aufzählung der Quindecimviri wird ausdrücklich mit $H A E C$ OMNIA PERACTA SVNT XVIvir(is) s(acris) f(aciundis) ... eingeleitet, und es leuchtet nicht ein, warum ein Mitglied des Kollegiums nicht in dieser Aufzählung erwähnt sein soll. Das Kollegium, dem die einmalige Aufgabe zugefallen ist, einen Anlass wie die Säkularspiele durchzuführen, musste vollständig der Nachwelt überliefert werden.

dazu auch Gagé (1955) 702 und Hanslik RE IX A, I (I96I) i I 2 ff. s. v. Vinicius Nr. 6. Auch Syme (I986) 49 geht von einem Quindecimvir der Säkularspiele dieses Namens aus. 